CA7-05:

\section{Transforming Primary Care in 3 NCQA-Certified PCMHs}

Ming Tai-Seale ${ }^{1}$; Daniel Dohan ${ }^{2}$; Sukyung Chung ${ }^{1}$; Dorothy Hung ${ }^{1}$; Dominick Frosch $^{1}$; Caroline Wilson ${ }^{1}$; Mary McCuistion ${ }^{1}$; Nidhi Kohli ${ }^{1}$; Cheryl Stults ${ }^{1}$; Harold Luft

${ }^{1}$ Palo Alto Medical Foundation, Sutter Health; ${ }^{2}$ University of California San Francisco

Background/Aims: Hopes are high for revitalizing primary care through transformation to a Patient- Centered Medical Home (PCMH) model. This model has been implemented in some capitated integrated delivery systems, but the transferability of PCMH to practices with a mixture of fee-for- service (FFS) and capitation contracts is uncertain. This study documents and analyzes how a transformation into a PCMH was achieved in a largely FFS multispecialty group practice in Northern California which has 30 clinics in three geographically distinct divisions. Division A was certified by NCQA at level 3 PCMH, Divisions B and C at level 2 PCMH. Methods: Multi-method case study includes qualitative data from semi-structured key informant interviews and quantitative data from medical records and administrative sources in 2005-2010. Key informants were purposefully sampled to capture experiences in all divisions and included executives, frontline physicians, nurse, health educators and medical assistants. We report on the interviews that were audio recorded, transcribed and analyzed thematically. We also present some initial quantitative findings on the association between the levels of NCQA PCMH certification and measures of process and outcomes of care. Results: Thirty-three key informant interviews have been completed to date. Four emerging themes are: [1] quality improvement efforts and responses to local market pressures pre-dated the national focus on $\mathrm{PCMH}$; [2] successful implementation of some PCMH components occurred in a top-down fashion with physician champions; [3] dominant business line (FFS) presents disincentive for some PCMH components; and [4] organizational culture differences may affect how well certain initiatives are taken up and sustained. EHR data reveal that the level 3 PCMH division had the shortest wait time to a 3 rd next available appointment for a longer visit (i.e., improved access), the highest proportion of patients with diabetes having their HbA1c measured every 6 months (improved process) and the highest proportion of patients with diabetes with their blood pressure under control (130/80) (improved outcomes). Discussion: We conclude that implementation of many PCMH components pre-dated the national focus on PCMH. Successfully implemented elements had organizational champions in practice cultures supportive of innovations. Some clinical process and outcome performance are consistent with the level of NCQA certification.

Keywords: Medical Home; Primary Care; Health Services Research doi:10.3121/cmr.2012.1100.ca7-05

\section{Healthcare Disparities}

PS2-01:

DISparities and Culture: Outcomes in Vitality Research (DISCOVeR) Program

Ariel Holland ${ }^{1}$; Kristen Azar ${ }^{1}$; Elsie Wang ${ }^{1}$; Eric Wong ${ }^{1}$; Beinan Zhao ${ }^{1}$; Rocio Morales $^{1}$; Latha Palaniappan ${ }^{1}$

${ }^{1}$ Palo Alto Medical Foundation, Sutter Health

Background/Aims: National health priorities include reduction of health disparities among racial/ethnic minorities. The cultural and linguistic diversity in the Palo Alto Medical Foundation (PAMF) service areas presented a compelling case for improving the collection of race, ethnicity, ancestry and primary language $(\mathrm{R} / \mathrm{E} / \mathrm{A} / \mathrm{L})$ data to accurately assess and address health disparities across all racial/ethnic groups. Methods: In May 2008, the PAMF began collecting patient self-reported R/E/A/L information, following the format of the U.S. 2000 and 2010 Census. The paper questionnaire (available in 9 languages) collected information on race (including Asian subcategories), Hispanic Origin (including Hispanic subcategories), ancestry (free text), preferred spoken language (free text), and interpreter services ( $\mathrm{Y}$ or $\mathrm{N}$ ). This information was subsequently entered in the patient's electronic health record by clinic staff. Results: Across
PAMF, there are over 650,000 active patients with approximately 2.3 million patient visits per year. To date, of the active PAMF patients who have selfreported $\mathrm{R} / \mathrm{E} / \mathrm{A} / \mathrm{L}$ information (65\% of all active patients), $54 \%$ self-identify as Non-Hispanic White/Caucasian, 30\% self-identify as one of the 6 major Asian racial/ethnic groups (12\% Asian Indian, 11\% Chinese, 3\% Filipino, $1 \%$ each of Japanese, Korean and Vietnamese), 2\% self-identify as Black/ African American and approximately 10\% self-identify as Hispanic/Latino. Most patients are willing to provide the requested information $(>90 \%)$. The accuracy of transfer of data from the paper questionnaire to the EHR across questions was high (92-97\%). Discussion: Patient R/E/A/L data has been used to identify racial/ethnic differences in cardiovascular disease and type 2 diabetes prevalence rates. These research findings have allowed PAMF to implement several culturally competent clinical programs, with the goal of improving healthcare for diverse populations. Our experience demonstrates the feasibility and benefits of collecting $\mathrm{R} / \mathrm{E} / \mathrm{A} / \mathrm{L}$ data in an ambulatory health care setting to assess and address health disparities.

Keywords: Race; Ethnicity; Healthcare Disparities

doi:10.3121/cmr.2012.1100.ps2-01

PS2-02:

Reducing Higher Prevalence of Diabetes Mellitus Among South Asian Populations in Canada

Syeda Kabir ${ }^{1}$

${ }^{1}$ York University, Toronto, Canada

Background/Aims: Statistical evidence confirms a disparity in diabetes mellitus prevalence by ethnicity in Canada. Diabetes mellitus (DM) prevalence was $6.4 \%$ of total populations for 2010 in Canada (PHAC, 2011). Canadian Diabetes Association (2006) reports that South Asian populations in Canada are at a greater risk of having DM. While DM prevalence in Canada was at 5.2\% for total populations in 2005, it was almost three folds higher, at $14 \%$ for South Asian populations. Canada is a world leader in scholarship of social determinants of health (SDH). However a gap between knowledge and action in SDH is evident in policies and practices of the Canadian government. Expert Panel Report (PHAC, 2009) contends that increasing prevalence of DM poses significant threats to the sustainability of the public health care system in Canada. With increased disparity in population health outcome it is imperative to investigate alternative approaches to policies and practices that will effectively lessen the prevalence of DM among South Asian populations in Canada. In this paper, I study the dominant approach in health organizations that inform/formulate/direct policies and practices, to reduce prevalence and/or to delay the onset of DM among South Asian populations in Canada. Methods: In this paper I advance my investigation with a political economy lens of social determinants of health. For qualitative inquiry I analyzed primarily text based documents from the organizations mentioned in the results section. I collected quantitative data from the World Health Organization, the Organization of Economic Co-operation and Development, the Public Health Agency of Canada, and Health Canada, between the time periods of 1998 and 2011. Results: Health Canada, Public Health Agency of Canada, Ontario Ministry of Health and Long Term Care, Ontario Ministry of Health Promotion, and Diabetes Association of Canada, have a dominant medical model approach in policies and practices to reduce the prevalence of T2DM among South Asian populations in Canada. Discussion: To reduce disparity in health outcome and to lessen DM prevalence among South Asian populations in Canada, it is imperative to put into effect a social determinants of health approach towards healthy public policies in Canada.

Keywords: Diabetes Prevalence; Social Determinants of Health; Healthcare Disparities

doi:10.3121/cmr.2012.1100.ps2-02 